assess the ability of ambulance staff to achieve successful outcomes using existing training.

All the patients included in the study were emergency cases-that is, either 999 calls or cases classed as an emergency by a general practitioner-and all had "hands on" assessment by ambulance and medical staff. To suggest that this prospectively performed study was a "table top theoretical exercise" is therefore far from the truth.

All of the patients admitted to a resuscitation area were assessed in detail, not merely the 396 who died. The patients in resuscitation areas constituted $4 \cdot 6 \%$ of all the emergency cases studied in Edinburgh and $4.3 \%$ of the Glasgow cases. These figures are similar to those reported for accident and emergency departments elsewhere in Britain.

Though "abundant evidence in favour of extended ambulance skills" exists in the United States, the situation in Britain is very different. Drs Baskett and Sleet claim that "hard data" are available from centres that have had years of experience with ambulancemen trained to paramedic level. We do not know of studies of this kind that compare basic and advanced ambulance skills, and we note that Drs Baskett and Sleet do not give references for such results.

As we indicated in our paper, the benefits of advanced training will be seen mainly in patients requiring cardiopulmonary resuscitation. We do not dispute the role of early defibrillation for ventricular fibrillation, and we agree with $\mathrm{Mr}$ William Rutherford (28 February, p 578) that there is a strong case for concentrating resources in this area. In the absence of cardiopulmonary resuscitation by a bystander, however, the limiting factor is the time the ambulance service takes to respond. We refute the comment that "the journey times . . leave something to be desired." The time referred to as "journey time" in fact represents the time from the initial 999 call to arrival in hospital and not the response time of the ambulance service.

We in no way denigrated the fact that "only 54" lives might have been saved; this figure represented the maximum possible benefit, and to suggest that over 2500 lives could be saved in Britain every year is an inappropriate interpretation of our results. As the authors indicate, we took no account of the effect of extended training on morbidity. We would be delighted to hear of a scientific and reproducible assessment fo morbidity in patients before they reach hospital.

JAMES D URQUHART

\begin{abstract}
Information Services Division,
Common Services Agency,
\end{abstract}

Edinburgh EH5 3SQ

\section{Phototherapy and dithranol treatment of psoriasis}

SIR,-Dr P M Farr and coworkers (24 January, p 205) attempted to define whether ultraviolet treatment hastened the improvement in psoriasis treated by dithranol. They studied the response to three different types of apparatus: a medium pressure mercury arc lamp, an array of six $60 \mathrm{~cm}$ fluorescent sunlamps, and an array of six $60 \mathrm{~cm}$ ultraviolet fluorescent sunlamps.

I agree that both ultraviolet radiation and dithranol are effective treatments for plaque psoriasis. ${ }^{12}$ Dr Farr and colleagues' tables II and III, however, contain clinical details that may have led to a premature conclusion about the efficacy of the different types of ultraviolet equipment studied.
Table II shows that the lesions on the nonirradiated, dithranol treated (control) arms in the patients treated with the mercury arc lamp took only 7.5 days to reach half of their initial thickness This compared with nine days for the two other ultraviolet treatment groups.

Similarly, table III showed that the mean clearance time for the lesions on the non-irradiated arm in patients treated with the mercury arc lamp was 13.5 days, compared with 17 and 18 days, respectively, for the control arms of the patients treated with the fluorescent sun lamp and Helarium. The mean time to clearance in the irradiated arms was 13.5 days for the mercury arc lamp, 14 days for the fluorescent sunlamp tubed lamps, and 12.5 days for the Helarium lamps.

One explanation may be that the rate of clearance of psoriasis achieved with dithranol in the patients treated with the nercury arc lamp could not be improved by additional ultraviolet, whereas the psoriasis in the patients treated with the Helarium lamps and fluorescent sunlamps was clearing at a slower rate and thus the ultraviolet seemed to produce better results.

Though I accept Dr Farr and coworkers' suggestions that improved lamps are indeed desirable for psoriasis phototherapy, I suggest that further research is needed, with randomisation of patients to the different treatment categories to permit more accurate evaluation of the potential benefits of different ultraviolet lamps.

In addition, a more suitable study would measure the effects of treatment with ultraviolet radiation and emollients alone using the differen ultraviolet lamps described. ${ }^{1}$ Dr Farr and colleagues have shown clearly the efficacy of dithranol alone in the treatment of psoriasis.

\section{Nicholas J Low}

UCLA School of Medicine,

Los Angeles,

CA 90024 ,

United States

1 Lowe NJ, Wortzman M, Breeding J, et al. Coal tar phototherapy for psoriasis re-evaluated: erythemogenic versus suberythemogenic ultraviolet with a tar extract in oil and crude coal tar. 7 Am Acad Dermatol 1983;8:781-9.

Lowe NJ, Ashton RE, Koudsi H, Verschoore M, Schaeffer $H$ Anthralin for psoriasis: short contact anthralin therapy compared with topical steroid and conventional anthralin. $\mathcal{f} A m$ Acad Dermatol 1984;10:69-72.

\section{AIDS counselling and informed consent}

SIR,-Dr Alan B Shrank (14 February, p 445) objects to counselling patients before he requests a test for antibodies to the acquired immune deficiency syndrome (AIDS) and does not see this as part of good history taking. If he had seriously been considering a diagnosis of AIDS surely he should be thankful that the pathologist may have protected him, and perhaps his employing authority, from the consequences of taking the specimen without fully informed consent. If the patient had subsequently been found to be positive for the human immunodeficiency virus (HIV) and his wife seropositive and pregnant after the honeymoon would Dr Shrank have been judged negligent in having failed to advise against pregnancy until after the serological results for the husband were known, especially as pregnancy may precipitate clinical AIDS in the female carrier of the virus as well as probably infecting the fetus?

Unlike Dr Shrank I have coped with a clinically well bisexual patient who, unfortunately, when tested elsewhere was found to be positive for antibodies to HIV but had no knowledge that the test was being performed until he was presented with the result. Fortunately, this patient did not commit suicide, unlike some HIV positive patients, and he remained clinically well and had not infected his wife or child. This episode has reinforced my conviction that counselling before as well as after the test is in the patient's interests, and only very exceptionally can it legitimately be omitted. The council of the BMA endorses this view, ${ }^{1}$ as does the Department of Health and Social Security, whose representative has added (in a personal communication) an important note: "Although it may have been common practice in the past to test patients for all sorts of diseases without specific consent, the advent of AIDS is causing the profession to question the wisdom (and legality) of such practices."

May we also set aside the spurious argument that compares patients positive for antibodies to HIV to patients with cancer because AIDS is, like some forms of cancer, invariably fatal; how often are patients with cancer put at risk of losing their homes and jobs as a result of innuendo? Fortunately, some doctors acknowledge that they have sexual "hang ups" that preclude them from discussing frankly and with confidence and sympathetic understanding sexual questions with the patient that need cause neither embarrassment nor offence to either party. Hopefully, more doctors will follow their peers and attend training in AIDS counselling; they will then be in a better position to help their patients.

C A MORRIS

Shrewsbury SY2 6PB

1 BMA. Third statement on AIDS. London: BMA. 1987.

Tennis elbow: conservative, surgical, and manipulative treatment

SIR,-There is a very simple cure for many patients with tennis elbow, which is little known and which is only briefly hinted at by $\mathrm{Mr}$ Thomas G Wadsworth (7 March, p 621).

Some years ago, when I played a great deal of tennis, I developed the typical lateral elbow in my right arm. This steadily worsened and was relieved for a day or two only after cortisone injections by an orthopaedic colleague. Rest gave no more than temporary relief, and I began to think that I should stop playing tennis. I mentioned this intention when operating at a cottage hospital one day. A general practitioner in the theatre, $\operatorname{Dr} M \mathrm{R}$ Sheridan, bluntly responded: "Rubbish! Go to Briggs's in Wood Green high street and get yourself a racquet with a bigger handle." I took the doctor's advice and went to Briggs's shop as soon as I finished operating and bought a new racquet with a handle $3.75 \mathrm{~cm}$ larger in circumference than my existing one.

A day or two later I played three sets of tennis with Dr Sheridan. On taking my first service, I said: "I shall never be able to serve with this large handle." My medical advisor retorted: "Shut up! You'll be alright by the end of the first set." $\mathrm{He}$ was absolutely right. Within half an hour I had adjusted my grip and never had any recurrence of trouble in the next 30 years. Lateral elbow pain is also often caused by gripping too small handles on shears and other garden tools. This can be prevented by building up the handles to the requisite thickness, and I have helped many sufferers to cure themselves in this way.

The mechanism of relief is simply explained if the position of the wrist when the hand is gripping objects of different thicknesses is studied. The smaller the circumference of the object held, the less the degree of dorsiflexion of the wrist. As progressively thicker objects are held dorsiflexion increases, reducing the tension on the forearm extensor muscle origins. It seems that with a 